\title{
HEIDEGGERS REZEPTION DES TAOISMUS: DIE NOTWENDIGKEIT DES UNNÖTIGEN (無用之用) IN DER LEISTUNGSGESELLSCHAFT
}

\author{
Choong-Su Han* \\ choongsu.han@gmail.com
}

\begin{abstract}
IN GERMAN Der vorliegende Beitrag widmet sich zwei Schriften Heideggers, die bisher kaum untersucht worden sind, nämlich Die Einzigkeit des Dichters (1943) und Abendgespräch in einem Kriegsgefangenenlager in Rußland zwischen einem Jüngeren und einem Älteren (1945). Diese Schriften sind vor allem insofern bemerkenswert, als der taoistische Gedanke der Notwendigkeit des Unnötigen (無用之用, wuyongzhiyong) in ihrem Zentrum steht. Heideggers Rezeption dieses Gedankens ist, wie im vorliegenden Beitrag gezeigt wird, aber keine bloße- Aufnahme, sondern vielmehr eine schöpferische Aneignung des ostasiatischen Gedankengutes für sein eigenes Seinsdenken. Zunächst wird Heideggers Auseinandersetzung mit dem Gedanken der Notwendigkeit des Unnötigen dargestellt. Danach wird versucht, im Anschluss an Heideggers Kunstphilosophie darüber nachzudenken, inwiefern das Kunstwerk als das Unnötige in der Leistungsgesellschaft notwendig ist.
\end{abstract}

Schlüsselwörter: die Notwendigkeit des Unnötigen, Heidegger, Laozi, Zhuangzi, Leistungsgesellschaft, Kunst

Abstract in English: The present contribution analyses two writings of Heidegger which have almost never been examined until now: The Uniqueness of the Poet (1943) and Evening Conversation: In a Prisoner of War Camp in Russia between a Younger and an Older Man (1945). These writings are very

1 Department of Philosophy, Ewha Womans University. Artigo recebido em 24/05/2016 e aprovado em $26 / 03 / 2017$. 
important for the comparative study of Heidegger's philosophy and East Asian thinking, not least because they are based on the Taoist idea of the necessity of the unnecessary (無用之用, wuyongzhiyong). Heidegger's reception of this idea is, as shown in this contribution, not simply a bare adoption, but rather a creative appropriation of East Asian thought for his own thinking of Being. First, this contribution shows Heidegger's confrontation with the idea of wuyongzhiyong. It then attempts to reflect on the extent to which the artwork, as that which seems to be useless, is necessary in a results-oriented society by looking at Heidegger's philosophy of art.

Keywords: the necessity of the unnecessary, Heidegger, Lao-Tzu, Chuang$T z u$, a result-oriented society, art

\section{Heidegger und die Schrift Tao Te King}

Es ist bekannt, dass sich Heidegger mit dem taoistischen Denker Laozi ( 老子) auseinandergesetzt hat. Eine explizite Auseinandersetzung findet sich in dem Vortrag Das Wesen der Sprache (1957/58). Dort unternimmt Heidegger den Versuch, ,uns [die Zuhörer des Vortrages] vor eine Möglichkeit zu bringen, mit der Sprache eine Erfahrung zu machen“. (Heidegger, 1985, p. 185). Dabei untersucht er die Nachbarschaft von Dichten und Denken, indem er sich zwischen ihnen hin und her bewegt. Heidegger wird aufmerksam auf diese Bewegung und richtet sein Augenmerk auf das Wort „Weg“. Im Anschluss daran erwähnt und erklärt er das Wort „Tao“ folgendermaßen: „Das Leitwort im dichtenden Denken des Laotse [老子] lautet Tao und bedeutet, eigentlich“ Weg." (Heidegger, 1985, p. 187). Anscheinend kennt sich Heidegger mit dem Taoismus und der altchinesischen Sprache gut aus.

Bereits früher hat er sich auf implizite Weise mit der taoistischen Schrift Tao Te King (道德經) beschäftigt, und zwar im Vortrag Einblick in das was ist (1949). Die Beschäftigung kennzeichnen wir ${ }^{1}$ als implizit, denn dort liegt kein Hinweis auf den Taoismus vor. Am Anfang des Vortrages wird problematisiert, dass ,trotz allem Überwinden der Entfernungen die Nähe dessen, was ist, ausbleibt“‘. (Heidegger, 1994, p. 4). Heidegger zufolge lässt sich die Nähe mit dem in der Nähe Seienden vorfinden. Daher führt er einen Gebrauchsgegenstand (Krug) als Beispiel an, um dadurch das Wesen der Nähe zu erfahren. Bevor 
Heidegger auf das Phänomen der Nähe eingeht, untersucht und bestimmt er das Wesen des Kruges mit folgenden Worten: „Die Leere, dieses Nichts am Krug, ist das, was der Krug als das fassende Gefäß ist.“ (Heidegger, 1994, p. 7-8). Nirgendwo im Kontext dieser Aussage beruft sich Heidegger auf Laozi oder Zhuangzi (莊子). Daher hat seine Wesensbestimmung des Kruges als Leere scheinbar nichts mit dem Taoismus zu tun.

Aber diejenigen, denen das ostasiatische Denken vertraut ist, verstehen unschwer, dass der Ausdruck „Nichts am Krug“ aus dem elften Kapitel der Schrift Tao Te King stammt. In diesem Kapitel geht es um den Gedanken, dass „was nicht ist [無]“, „Zum Werk“ „dient“. (Laotse, 1919, p. 13). Dieser Gedanke wird dort mit drei Beispielen veranschaulicht, nämlich mit „Wagen“, „Topf [器]“ und „Kammer“. Das altchinesische Zeichen, 器“ bedeutet aber auch „Krug“, „Gerät“ und „Werkzeug“. Die Stelle, die für Heideggers Wesensbestimmung des Kruges relevant ist, übersetzt Richard Wilhelm folgendermaßen ins Deutsche: „Man höhlet Ton und bildet ihn zu Töpfen: In ihrem Nichts [無] besteht der Töpfe [器] Werk.“ (Laotse, 1919, p. 13). Damit meint Laozi ebenfalls, dass die Höhle (wortwörtlich übersetzt: das Nichts) den Topf nutzbar macht. Wenn die Nützlichkeit das Wesen des Gebrauchsgegenstandes ausmacht, dann besteht das Wesen des Topfes eigentlich in dessen Nichts. Dementsprechend bestimmen sowohl Laozi als auch Heidegger das Wesen von 器 (Topf bzw. Krug) als dessen Nichts (無). 2

\section{Fragestellung und Skizze des vorliegenden Beitrages}

Der Vortrag Einblick in das was ist bezieht sich offenbar nicht auf die Schrift Tao Te King. Daher stellt sich folgende Frage, ob Heidegger seine Wesensbestimmung des Kruges wirklich aus dem Taoismus übernommen hat. Unsere Antwort auf diese Frage ist positiv. Um sie zu begründen, werden wir zwei Schriften Heideggers betrachten, denen jeweils ein taoistischer Gedanke zugrunde liegt. Die erste Schrift heißt Die Einzigkeit des Dichters (1943) und ist für die interkulturelle Heidegger-Forschung insofern entscheidend, als der ganze Text des elften Kapitels der Schrift Tao Te King dort zitiert ist. Dabei gibt Heidegger die Quelle des Zitats ausdrücklich an. (Heidegger, 2000, p. 43). Die Schrift Die Einzigkeit des Dichters weist also eine eindeutige Verbindung zwischen Heidegger und Laozi nach. Die zweite Schrift heißt Abendgespräch in einem Kriegsgefangenenlager in Rußland zwischen einem Jüngeren und einem Älteren (1945) ${ }^{3}$ und beinhaltet Zhuangzis Worte, deren Quelle aber nicht

2 Reinhard May weist darauf hin, dass Heideggers Wesensbestimmung des Kruges als Leere unter dem Einfluss des elften Kapitels von Tao Te King steht. (May, Tezuka, 1996, p. 30). Auch Kah Kyung Cho sagt, dass die Leere des Kruges an die Zeilen von Laozi anklingt. (Cho, 1987, p. 92). 
klar angegeben ist. Bevor sie ein Gesprächspartner anführt, sagt er: „Aber zur guten Nacht und vielleicht auch zum Dank möchte ich [der Ältere] Dir [dem Jüngeren] doch sogleich noch ein kurzes Gespräch zweier Denker erzählen, das ich mir in meiner Studentenzeit aus einer historischen Darstellung der chinesischen Philosophie abgeschrieben habe [...]. Die Namen der beiden Denker sind mir entfallen." (Heidegger, 1995, p. 239). Aber für diejenigen, die sich mit dem Taoismus gut auskennen, ist offenkundig, dass das zitierte Gespräch aus dem 26. Kapitel der Schrift Zhuangzi (莊子) stammt. (Dschuang Dsï, 1972, p. 281). Da es eine grundlegende Rolle in der Abendgespräch-Schrift spielt, steht diese unter dem großen Einfluss von Zhuangzi.

Diese beiden Schriften Heideggers sind im Hinblick auf das ostasiatische Denken noch kaum untersucht worden. ${ }^{4}$ Der Grund für diese Unbekanntheit liegt wohl darin, dass sie jeweils erst vor relativ kurzer Zeit erschienen sind. Die Schrift Die Einzigkeit des Dichters gehört zum Band Zu Hölderlin/Griechenlandreisen, der erst im Jahr 2000 veröffentlicht wurde. Die Abendgespräch-Schrift gehört zum Band Feldweg-Gespräche (1944/45). Dieser Band besteht aus drei fiktiven Gesprächen. Einen Teil des ersten Gespräches publizierte Heidegger unter dem Namen Gelassenheit bereits im Jahr 1959. Das zweite und das dritte Gespräch erschienen im Jahr 1995. Außerdem wurde der Band Feldweg-Gespräche erst im Jahr 2010 ins Englische übersetzt. (Heidegger, 2010).

Zunächst werden wir Heideggers Rezeption des Taoismus darstellen, und zwar aufgrund der Schriften Die Einzigkeit des Dichters und Abendgespräch in einem Kriegsgefangenenlager in Rußland zwischen einem Jüngeren und einem Älteren. Diese Darstellung wird die beiden Schriften, die bisher unberührt sind, für Heidegger-Forschung zugänglich machen. Dabei werden wir uns klarmachen, dass seine Rezeption keine bloße Aufnahme, sondern insofern eine schöpferische Aneignung des ostasiatischen Gedankengutes ist, als er im Anschluss an Zhuangzi die „ungehemmte Leistungssteigerung“ einer radikalen Kritik unterzieht. (Heidegger, 1995, p. 211). So überbrückt Heidegger eine zeitlich und räumlich große Entfernung zwischen der antiken östlichen Denktradition und der modernen westlichen Leistungsgesellschaft. Zum Schluss werden wir im Anschluss an Heidegger darüber nachdenken, insofern die Kunst als das Unnötige in der Leistungsgesellschaft notwendig ist.

fangenenlager in Rußland zwischen einem Jüngeren und einem Älteren die Abendgespräch-Schrift. sing Paths In-Between, Albany: State University of New York Press 2006; Günter Wohlfart, Der philosophische Daoismus: philosophische Untersuchungen zu Grundbegriffen und komparative Studien mit besonderer Berücksichtigung des Laozi (Lao-tse), Köln: Edition Chora 2001; Lin Ma/Jaap van Brakel, „Heidegger's Comportment Toward East-West Dialogue“, Philosophy East and West, 56 (4), 2006, 519-566. 


\section{Tao Te King und Die Einzigkeit des Dichters}

Die Schrift Die Einzigkeit des Dichters beginnt damit, im Hinblick auf das Wesen der Dichtung zwischen historischem Nachdenken und geschichtlicher Besinnung zu unterscheiden. Mit dem historischen Nachdenken meint Heidegger, dass die Dichter untereinander verglichen und damit gleichgemacht werden; im Gegensatz dazu beachtet die geschichtliche Besinnung das „Gedichtete“, denn ,[d]er Dichter selbst sagt es, was Dichtung ist"; für Heidegger ist dieser Dichter Hölderlin. (Heidegger, 2000, pp. 35-37).

Für die Besinnung auf Hölderlins Dichtung bedarf es Heidegger zufolge der Übung, die darin besteht, die „Achtsamkeit“" zu ,lernen“; demnach stellt sich folgende Frage: ,Wie lernen wir aber die Achtsamkeit, wir, die Spätgeborenen einer seit Jahrhunderten währenden Achtlosigkeit?“ (Heidegger, 2000, p. 42). Darauf antwortet Heidegger: „Wir lernen die Achtsamkeit, indem wir in das unscheinbare Einfache blicken, es je und je ursprünglicher aneignen und vor ihm scheuer und scheuer werden." (Heidegger, 2000, p. 42-43). Da das unscheinbare Einfache schlicht und unauffällig ist, macht es uns keinen besonderen Eindruck. Wir müssen uns darum bemühen, es anzuschauen. Dabei erlernen wir die Achtsamkeit. Mit dem „unscheinbaren Einfachen“ ist das „Sein“ gemeint, das ,im Unterschied zum Seienden“ genannt wird; in ,diesem Unterschied“"kommt das Sein, so Heidegger, auch bei Lao-Tse vor, deswegen wird der ganze ,elfte Spruch seines TaoTe-King“ zitiert. (Heidegger, 2000, p. 43).

Im Hinblick auf die oben zitierte Übersetzung Heideggers ist bemerkenswert, dass sie nicht mit der damaligen bekannten Übersetzung von Richard Wilhelm übereinstimmt. Die Stelle, die relevant für Heideggers Wesensbestimmung des Kruges und somit auch unsere Forschung ist, übersetzt Wilhelm folgendermaßen: „Man höhlet Ton und bildet ihn zu Töpfen: In ihrem Nichts [無] besteht [有] der Töpfe [器] Werk [用].“(Laotse, 1919, p. 13). Die Übersetzung, die in der Schrift Die Einzigkeit des Dichters vorkommt, lautet: „Aus dem Ton ent-stehen die Gefäße, / Aber das Leere [無] in ihnen gewährt das

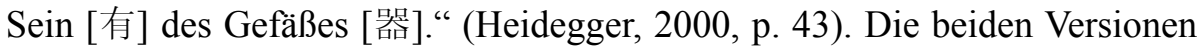
sind zwar nicht gleich, drücken aber denselben Gedanken aus, dass das Nichts und die Leere des Topfes oder Gefäßes dessen Nutzen bestehen/existieren/sein lässt (,當其無有器之用“). (노자, 2012, p. 43).

Die äußerliche Verschiedenheit zwischen den beiden Übersetzungen lässt uns folgende Frage stellen, welche oder wessen Übersetzung Heidegger zur Verfügung hatte. Oder sollten wir auch die Möglichkeit in Erwägung ziehen, dass er das elfte Kapitel der Schrift Tao Te King vielleicht selbst ins Deutsche übersetzen könnte? Es ist bekannt, dass Heidegger mit dem Sinologen Paul Shih-Yi Hsiao den Versuch unternahm, diese Schrift aus dem Altchinesischen 
ins Deutsche zu übersetzen; aber nicht alle Kapitel, sondern nur die ersten achten Kapitel stellten sie fertig. (Neske, 1977, p. 326). Diesem Bericht zufolge gehört das elfte Kapitel zwar nicht zu den übersetzten Kapiteln. Er gibt aber Heideggers Interesse daran zu erkennen, die Schrift Tao Te King persönlich weiter zu übersetzen. Demnach kann die Möglichkeit nicht ausgeschlossen werden, dass auch die in der Schrift Die Einzigkeit des Dichters angeführte Übersetzung von Heidegger selbst gemacht worden wäre.

In der Übersetzung Heideggers sind zwei Wörter bemerkenswert, nämlich „Leere“ und „Gefäß“, und zwar zunächst deswegen, weil sie nicht in der Übersetzung Wilhelms vorkommen. Außerdem fungieren sie als Grundbegriffe im Vortrag Einblick in das was ist, den Heidegger sechs Jahre nach der Abfassung der Schrift Die Einzigkeit des Dichters hielt. (Heidegger, 1994, pp. 7-8). Daraus ergibt sich klar, dass die dem besagten Vortrag zugrunde liegende Wesensbestimmung des Gefäßes als Leere unter dem Einfluss des elften Kapitels der Schrift Tao Te King steht. Ein weiteres Wort „Sein“ zieht unsere Aufmerksamkeit auf sich. Dieses Wort ist die Übersetzung des altchinesischen Zeichens ,,有“, das viele Bedeutungen hat: unter anderem „sein“, „haben“, „,besitzen“", „vorhanden sein“, „,existieren“, „,vorliegen“, „,bestehen“. Während Wilhelm dieses Zeichen mit „bestehen“ übersetzt, ist es bei Heidegger mit „Sein“ wiedergegeben. Heideggers Wortwahl ist einerseits eine einfache und treue Übersetzung, weil „,das Sein“ oder, genauer gesagt, „,zu sein“ die Grundbedeutung von 有 ist. Andererseits ist sie insofern eine kreative Übersetzung, als Heidegger mit dieser Wortwahl Laozis Denken in Verbindung mit dem eigenen Seinsdenken bringt. Demnach ist Heideggers Rezeption der Schrift Tao Te King auch eine schöpferische Aneignung des Taoismus.

Um die Behauptung zu untermauern, dass die taoistischen Gedanken durch das und im Denken Heideggers variiert werden, ziehen wir eine weitere Schrift heran, namentlich Abendgespräch in einem Kriegsgefangenenlager in Ru $\beta$ land zwischen einem Jüngeren und einem Älteren. Diese Schrift wurde zwei Jahre nach der Entstehung der Schrift Die Einzigkeit des Dichters verfasst. In diesen zwei Jahren geschah eine Veränderung mit Heideggers Denken. Als er das elfte Kapitel der Schrift Tao Te King in seiner Schrift Die Einzigkeit des Dichters übersetzte, überging er dabei das altchinesische Zeichen, 用“ (benutzen), das Wilhelm mit „Werk“ übersetzte. Aber in der Abendgespräch-Schrift spielt dieses Zeichen eine grundlegende Rolle.

4. Zhuangzi und Abendgespräch in einem Kriegsgefangenenlager in Rußland zwischen einem Jüngeren und einem Älteren

Im Zentrum der Abendgespräch-Schrift steht der Gedanke der ,Notwendigkeit des Unnötigen“. Er kommt erstmals in den Worten des Jüngeren vor: „Erst wer die Notwendigkeit des Unnötigen wissen gelernt hat, kann ungefähr 
wenigstens den Schmerz ermessen, der damit entspringt, daß dem Menschen das Denken verwehrt ist." (Heidegger, 1995, p. 221). Der Jüngere offenbart jedoch nicht, woher er den Ausdruck „die Notwendigkeit des Unnötigen“" genommen hat. Erst am Ende des Gespräches wird angedeutet, dass dieser Ausdruck etwas mit der altchinesischen Philosophie zu tun hat. (Heidegger, 1995, p. 239). Der Ältere führt nämlich einen kurzen Dialog zwischen zwei altchinesischen Denkern an, dessen Lehre darin bestehe, dass das Unnötige notwendig sei. Da ihre Namen aus seinem Gedächtnis angeblich geschwunden sind, bleibt es unbekannt, woher der Dialog stammt. Aber für diejenigen, denen der Taoismus vertraut ist, liegt es klar vor Augen, dass der Dialog seinen Ursprung in der Schrift Zhuangzi hat. (장자, 1999, p. 411). ${ }^{5}$

\section{Die von Heidegger angeführte Übersetzung lautet:}

Der Eine sagte: ,Ihr redet vom Unnötigen“. / Der Andere sprach: ,Erst muß einer das Unnötige erkennen, ehe man mit ihm vom Nötigen reden kann. Die Erde ist ja weit und groß, und doch braucht der Mensch, um zu stehen, nur so viel Platz, daß er seinen Fuß darauf setzen kann. Wenn aber unmittelbar neben dem Fuß ein Riß entstände bis hinab zu der Unterwelt, wäre ihm dann der Platz, worauf er steht, noch zu etwas nütze?` / Der Eine sprach: ,Er wäre ihm nichts mehr nütze‘. / Der Andere sprach: ,Daraus ergibt sich klar die Notwendigkeit des Unnötigen'. (Heidegger, 1995, p. 239).

Diese Übersetzung stimmt mit der Übersetzung Wilhelms überein. ${ }^{6} \mathrm{Au}-$ ßerdem versieht dieser den Dialog mit der Überschrift „Die Notwendigkeit des Unnötigen“. (Dschuang Dsï, 1972, p. 281). Demnach liegt die Vermutung nahe, dass Heidegger seinen Ausdruck ,die Notwendigkeit des Unnötigen“ aus der taoistischen Schrift Zhuangzi übernahm, und zwar mithilfe der Übersetzung Wilhelms. Was am Ende des Dialoges gesprochen wird, lautet im Original: ,然則無用之爲用也“. (장자, 1999, p. 411). Diese Worte werden abgekürzt,,無用之用“ (wuyongzhiyong) genannt. Das erste Zeichen, 無“ fungiert als Negation des nachfolgenden Zeichens. Das zweite Zeichen ,用“ bedeutet „,benutzen“ oder „Nutzen“. Das dritte Zeichen „之“ ist die Präposition, die die Zugehörigkeit des nachfolgenden Zeichens zum vorausliegenden Zeichen angibt. Das vierte Zeichen ist gleich dem zweiten. Dementsprechend kann der

5 In der ostasiatischen Antike musste keine Quellenangabe beim Zitieren der Klassiker gemacht werden, und zwar angeblich deswegen, weil sie jeder auswendig lernte.

6 Wilhelms Übersetzung lautet: „Hui Dsï sprach zu Dschuang Dsï: ,Ihr redet von Unnötigem.‘ / Dschuang Dsï sprach: ,Erst muß einer das Unnötige erkennen, ehe man mit inm vom Nötigen reden kann. Die Erde ist ja weit und groß, und doch braucht der Mensch, um zu stehen, nur soviel Platz, daß er seinen Fuß darauf setzen kann. Wenn aber unmittelbar neben dem Fuß ein Riß entstünde bis hinab zu der Unterwelt, wäre ihm dann der Platz, worauf er steht, noch zu etwas nütze?‘ / Hui Dsï sprach: ,Er wäre ihm nichts mehr nütze.' / Dschuang Dsï sprach: ,Daraus ergibt sich klar die Notwendigkeit des Unnötigen.“” (Dschuang Dsï, 1972, p. 281). 
taoistische Gedanke,,無用之用“ mit dem Ausdruck „Nutzen des Nutzlosen“ wiedergegeben werden. Der Ausdruck „Notwendigkeit des Unnötigen“ ist auch eine angemessene Übersetzung.

Um zu verstehen, was Heidegger mit diesem Ausdruck meint, betrachten wir die Abendgespräch-Schrift. Sie beginnt mit der Erzählung der morgendlichen Erfahrung mit „etwas Heilsame[m]“. (Heidegger, 1995, p. 205). Wer geheilt werden kann, muss vorher verletzt worden sein. Die Gesprächspartner, nämlich der Jüngere und der Ältere, sind verletzt und ,zerrissen“. (Heidegger, 1995, p. 206). Ihre „Wunde“ besteht darin, dass ihr Wesen ,aus der freien Weite des Denkens“",ausgestoßen“ ist; mit dieser Wunde meinen sie nicht den „Verzicht auf die Beschäftigung mit geistigen Sachen“, sondern vielmehr den „Entzug des Daseins, das auf dem Grunde des Denkens beruht“. (Heidegger, 1995, p. 219). Also wird ihnen das denkende Dasein (vita contemplativa) nicht mehr gegeben, deswegen leiden sie innerlich schwer: „Der brennende Schmerz ist, daß wir nicht für das Unnötige [das Denken] da sein durften.“ (Heidegger, 1995, p. 219).

Hierin sehen wir schon Heideggers Variation des taoistischen Gedankens. Dieser Gedanke wird in der Schrift Zhuangzi anhand verschiedener Beispiele (Menschen und Dingen) veranschaulicht. Für die Darstellung eines Beispiels ziehen wir ein Gespräch aus der Schrift Zhuangzi heran. In diesem Gespräch geht es um einen riesigen Baum, der so verwachsen und verkrüppelt ist, dass sich kein Tischler für ihn interessiert. Dieser Baum ist scheinbar zu nichts zu gebrauchen. Sein Besitzer beschwert sich bei Zhuangzi über diese Nutzlosigkeit. Dieser sagt aber, dass der Baum so lange hat leben können, gerade weil er eben keinen Nutzen gebracht hat. Zhuangzi fügt gleich hinzu, dass wir jedoch um den nutzlosen Baum herum spazieren gehen und in seinem Schatten ruhen können. (장자, 1999, pp. 53-54). Der Spaziergang und die Ruhe unterscheiden sich von der Arbeit, die unmittelbar Nutzen bringen soll. Aber ohne Pause wäre die alltägliche Arbeit nicht mehr möglich, geschweige denn die schöpferische. Die Auszeit ist also notwendig. Mit dem Gespräch über den scheinbar unnötigen Baum macht Zhunagzi seine Lehre der Notwendigkeit des Unnötigen anschaulich. ${ }^{7}$

Was Heidegger mit dem „Unnötigen“ in der Abendgespräch-Schrift meint, ist aber weder Mensch noch Ding, sondern das Denken. Dieses scheint unnötig zu sein, deswegen beschäftigen wir uns nicht mehr mit dem Denken. Vielmehr treiben wir viele angeblich wichtige Beschäftigungen (vita activa). Diese sind 
dem Jüngeren zufolge aber das „vermeintliche Nötige“, das „das Auge unseres Wesens blind für das Unnötige“ macht; der Ältere fügt gleich hinzu, „daß das Unnötige jederzeit das Nötigste von allem bleibt“. (Heidegger, 1995, p. 220). Im Anschluss daran spricht der Jüngere die Worte aus, die wir bereits oben erwähnt haben: „Erst wer die Notwendigkeit des Unnötigen wissen gelernt hat, kann ungefähr wenigstens den Schmerz ermessen, der damit entspringt, daß dem Menschen das Denken verwehrt ist.“ (Heidegger, 1995, p. 221). Diese Worte, in denen der taoistische Gedanke erstmals vorkommt, machen deutlich, dass Heidegger mit dem Unnötigen das Denken meint. „Das Denken ist somit das Unnötige“, sagt der Ältere. (Heidegger, 1995, p. 221).

Indem Heidegger das Denken das Unnötige nennt, eröffnet er die Möglichkeit, den taoistischen Gedanken in Verbindung zur Leistungsgesellschaft zu bringen, in der wir uns nämlich nur mit den scheinbar Nötigen beschäftigen. Diese Beschäftigung rottet ,etwas Wesenhaftes“ aus, damit es nicht mehr ,aufgehe und erblühe“; aber diese Ausrottung ,versteckt sich hinter einem Verfänglichen, das sich in der Gestalt der angeblich höchsten Menschheitsideale kundgibt"; eins dieser Ideale ist für Heidegger die ,ungehemmte Leistungssteigerung auf allen Gebieten des Schaffens“. (Heidegger, 1995, p. 211). In dieser Leistungsgesellschaft, ,[w]o also die Welt im Glanz des Aufstiegs, der Vorteile und der Glücksgüter erstrahlt", wird alles Seiende ,ins Nützliche“",verrechnet und eingerichtet“". (Heidegger, 1995, p. 236). Der Lauf der Leistungsgesellschaft kann durch das Unnötige gehemmt werden, weil dieses ihrem ,einzigen Gesetz“ widerspricht, das darin besteht, „,aß das Nötige das Nötigste und das allein Nötige sei“. (Heidegger, 1995, p. 236). Für die Überwindung der Leistungsgesellschaft ist das Unnötige (das Denken) also notwendig.

Die von Heidegger in der Abendgespräch-Schrift vorgenommene Variation des Taoismus bringt, wie gerade gezeigt, die antike östliche Denktradition und die moderne westliche (jetzt aber auch ostasiatische) Leistungsgesellschaft in innige Verbindung miteinander. Darüber hinaus erinnert uns seine Interpretation des Denkens als Unnötigen daran, dass er die Philosophie als das „unmittelbar nutzlose, gleichwohl herrschaftliche Wissen vom Wesen des Seienden" bezeichnet hat, und zwar in der Vorlesung Grundfragen der Philosophie. Ausgewählte „Probleme“ der ,Logik“, die er im Wintersemester 1937/38 an der Universität Freiburg hielt. (Heidegger, 1984, p. 2). Mit dieser Bezeichnung meint Heidegger, Philosophie nütze uns zwar unmittelbar nichts, weil sie „weder die Weltanschauung noch die Wissenschaft“ ersetzen könne; sie sei aber das „vorausspringende, neue Fragebereiche und Fragehinsichten eröffnende Wissen vom stets sich neu verbergenden Wesen der Dinge“. (Heidegger, 1984, p. 3). Die Philosophie wirkt also nur mittelbar, indem sie die Fragen nach dem Wesen des Dinges für die Wissenschaftler zugänglich macht. 
Dementsprechend können wir sagen, dass sie (das Unnötige) durchaus notwendig für die Erschließung der Wissenschaft ist. Heidegger veranschaulicht die Notwendigkeit des Unnötigen am Beispiel der Philosophie. Oder das Wesen der Philosophie kommt mithilfe des taoistischen Gedankens zum Vorschein.

Diese gedankliche Verwandtschaft zwischen Heidegger und Zhuangzi kann eine bemerkenswerte Koinzidenz sein. Oder sie besteht, weil sich Heidegger mit dem Taoismus auseinandergesetzt hat. Dann stellt sich folgende Frage, ob ihm die Schrift Zhuangzi bereits in den 1930 Jahren vertraut war. Auf diese Frage können wir noch nicht antworten, weil wir bisher nur die Schriften untersucht haben, die er in den 1940 Jahren verfasste: Die Einzigkeit des Dichters (1943), Abendgespräch in einem Kriegsgefangenenlager in Rußland zwischen einem Jüngeren und einem Älteren (1945) und Einblick in das was ist (1949). Die Antwort auf die Frage ist wohl positiv, weil darüber berichtet wurde, dass sich Heidegger auf ein Gespräch aus der Schrift Zhuangzi berief, und zwar im Jahr 1930, um damit die Intersubjektivität zu erklären. (Petzet, 1983, p. 24).

Diese frühere Rezeption des Taoismus ist bemerkenswert, weil sie uns dazu auffordert, nach weiteren gedanklichen Verwandtschaften zwischen Heidegger und Zhuangzi zu suchen. Aus einem Brief Heideggers an Elisabeth Blochmann geht hervor, dass die Vorarbeit für den Aufsatz Der Ursprung des Kunstwerkes in den Jahren 1931 und 1932 geleistet wurde, welche er überarbeitete, erweiterte und in den Jahren 1935 und 1936 vortrug. (Heidegger, Blochmann, 1989, p. 87). Offenbar widmete sich Heidegger der Besinnung auf das Wesen der Kunst bzw. des Kunstwerkes nach seiner Rezeption des Taoismus. Wir unternehmen den Versuch, seine Kunstphilosophie im Hinblick auf den taoistischen Gedanken der Notwendigkeit des Unnötigen zu betrachten.

\section{Kunst im Hinblick auf die Notwendigkeit des Unnötigen in der Leistungsgesellschaft}

Der Abendgespräch-Schrift zufolge haben wir keinen Zugang mehr zur freien Weite des Denkens. In diesem Ausgestoßensein besteht unsere Wunde. Wenn diese nicht eine körperliche, sondern eine metaphysische ist, dann kann sie durch die Kunst als ,eigentlich metaphysische Tätigkeit“ und „rettende, heilkundige Zauberin“" geheilt werden. (Nietzsche, 1980, p. 24, 57).

Die ungehemmte Leistungssteigerung war, wie oben gezeigt, ein Ideal, in deren Namen alles Seiende nur hinsichtlich seiner Nützlichkeit beurteilt, benutzt und verbraucht wurde. Dieses Ideal wird 70 Jahre nach der Abfassung der Abendgespräch-Schrift aber immer noch hochgehalten. Im Hinblick auf die Problematik der Leistungsgesellschaft macht Gernot Böhme folgende Bemerkung: „Wir leben in der BRD in einer Gesellschaft mit hervorragenden 
Einrichtungen [...]. Im Gegenteil sind alle Bürger im Stress und in einem Denken befangen, das ihnen auf allen Gebieten immer mehr Leistung abverlangt - und zwar nicht nur im Bereich der Arbeit, sondern auch in Konsum und Freizeit“. (Böhme, 2010, p. 7). Diese Diagnose bestätigt sich zurzeit auch in ostasiatischen Ländern.

Im Aufsatz Der Ursprung des Kunstwerkes erklärt Heidegger die Kunsterfahrung in zweierlei Hinsicht. Einerseits schaffen wir das Kunstwerk; andererseits bewahren wir es. Sowohl das Schaffen als auch das Bewahren stehen allerdings in einem engen Zusammenhang mit der Wahrheit des Seienden. Die bewahrende Erfahrung des Kunstwerkes ist insofern bemerkenswert, als es uns aus dem Gewöhnlichen heraus und in die „Offenheit“ (die Wahrheit) hinein rückt, die „durch es selbst eröffnet“" wurde. (Heidegger, 1977, p. 54). In dieser Offenheit stehen wir außerhalb des Nutzungszusammenhanges. Dabei verwandeln sich unsere ,gewohnten Bezüge zur Welt und zur Erde“, sodass wir uns „mit allem geläufigen Tun und Schätzen, Kennen und Blicken“ zurückhalten und ,in der im Werk geschehenden Wahrheit“ verweilen. (Heidegger, 1977, p. 54). Dieses ruhende Verweilen hat seine Grundlage im Wesen des Kunstwerkes, nämlich in der „Bestreitung des Streites zwischen Welt und Erde“; diese Bestreitung als die höchste Bewegtheit schließt ja die Ruhe nicht aus, sondern ein; diese dynamische Ruhe ermöglicht das „Insichruhen des Werkes“ und ferner unser Verweilen in seiner Offenheit (Lichtung). (Heidegger, 1977, p. 45). Die Bewahrung des Kunstwerkes ist zwar keine Tätigkeit, die unmittelbar Nutzen bringt, kann aber als Ruhepol in der Leistungsgesellschaft dienen. Wie der unnötige Baum den Schatten schenkt, so lässt uns das Kunstwerk auf seiner Lichtung verweilen.

Bisher haben wir Heideggers schöpferische Aneignung des Taoismus aufgezeigt und auf die gedankliche Verwandtschaft zwischen Heidegger und Zhuangzi hingewiesen. Daraus ergibt sich, dass wohl noch viele gedankliche Verwandtschaften zu entdecken bleiben. Das Ost-West-Gespräch ist für die Heidegger-Forschung noch lange nicht zu Ende, sondern hat erst gerade angefangen. Diese künftige Konversation wird keine bloße vergleichende historische Untersuchung sein, sondern sie wird als interkultureller Dialog zur Konversion der globalen Gesellschaft dienen.

\section{Bibliografie}

BÖHME, G. "Kritik der Leistungsgesellschaft". Bielefeld/Basel: Aisthesis-Verlag, 2010.

BUSSMANN, H. “Lexikon der Sprachwissenschaft”. Stuttgart: Kröner, 1983.

CHO, K. K. "Bewusstsein und Natursein. Phänomenologischer West-Ost-Diwan". Freiburg, München: Alber, 1987. 
DSCHUANG DSÏ, “Das wahre Buch vom südlichen Blütenland”. Translated into German by Richard Wilhelm. Düsseldorf, Köln: Diederichs, 1972.

FROESE, K. "Nietzsche, Heidegger, and Daoist Thought. Crossing Paths In-Between". Albany: State University of New York Press, 2006.

HEIDEGGER, M. "Der Ursprung des Kunstwerkes". In: "Holzwege" (GA5). Friedrich Wilhelm von Herrmann (Ed.). Frankfurt am Main: Vittorio Klostermann, 1977.

HEIDEGGER, M. "Grundfragen der Philosophie. Ausgewählte 'Probleme' der 'Logik" (GA 45). Friedrich Wilhelm von Herrmann (Ed.). Frankfurt am Main: Vittorio Klostermann, 1984.

HEIDEGGER, M. "Das Wesen der Sprache”. In: "Unterwegs zur Sprache” (GA12). Friedrich Wilhelm von Herrmann (Ed.). Frankfurt am Main: Vittorio Klostermann, 1985. HEIDEGGER, M. "Einblick in das was ist". In: "Bremer und Freiburger Vorträge" (GA79). Petra Jaeger (Ed.). Frankfurt am Main: Vittorio Klostermann, 1994.

HEIDEGGER, M. "Abendgespräch in einem Kriegsgefangenenlager in Rußland zwischen einem Jüngeren und einem Älteren”. In: "Feldweg-Gespräche” (GA 77). Ingrid Schüßler (Ed.). Frankfurt am Main: Vittorio Klostermann, 1995.

HEIDEGGER, M. "Die Einzigkeit des Dichters". In: "Zu Hölderlin. Griechenlandreisen” (GA 75). Curd Ochwadt (Ed.). Frankfurt am Main: Vittorio Klostermann, 2000.

HEIDEGGER, M. "Country Path Conversations". Translated into English by Bret W. Davis. Bloomington: Indiana University Press, 2010.

HEIDEGGER, M., BLOCHMANN, E. "Briefwechsel 1918-1968". Joachim W. Storck (Ed.). Marbach am Neckar: Deutsche Schillergesellschaft, 1989.

LAOTSE. “Tao Te King. Das Buch des Alten vom Sinn und Leben”. Translated into German by Richard Wilhelm. Jena: Diederichs, 1919.

MA, L., VAN BRAKEL, J. "Heidegger's Comportment Toward East-West Dialogue". In: Philosophy East and West, v. 56, n. 4, pp. 519-566, 2006.

MAY, R., TEZUKA, T., “Heidegger's Hidden Sources. East Asian Influences on his Work". Translated into English by Graham Parkes. London, New York: Routledge, 1996. NESKE, G. (Ed.). "Erinnerung an Martin Heidegger”. Pfullingen: Neske, 1977.

NIETZSCHE, F. "Die Geburt der Tragödie aus dem Geiste der Musik" (Kritische Studienausgabe, Band 1). Giorgio Colli, Mazzino Montinari (Ed.). Berlin/München: Deutscher Taschenbuch-Verlag, 1980.

PARKES, G. (Ed.). "Heidegger and Asian Thought". Honolulu: University of Hawaii Press, 1987.

PETZET, H. W. “Auf einen Stern zugehen. Begegnungen und Gespräche mit Martin Heidegger 1929-1976”. Frankfurt am Main: Societaets-Verlag, 1983.

WOHLFART, G. "Der philosophische Daoismus. Philosophische Untersuchungen zu Grundbegriffen und komparative Studien mit besonderer Berücksichtigung des Laozi (Lao-tse)". Köln: Edition Chora, 2001.

노자. “도덕경”. Translated into Korean by 오강남. 서울: 현암사, 2012.

장자, “장자". Translated into Korean by 오강남, 서울: 현암사, 1999. 